\title{
Non-Linear Abelian Scenarios and Yang-Mills Theory
}

\author{
S. Machado Moreira ${ }^{1}$, I.Soares ${ }^{2}$, D. Leite ${ }^{1}$, R. M. Doria ${ }^{2}$ \\ [1]Centro Brasileiro de Pesquisas Físicas, Rio de Janeiro RJ, 22290-180, Brazil \\ [2]Quarks, Petrópolis RJ, 25600, Brazil \\ saulomachado@gmail.com
}

\section{ABSTRACT}

We present a natural formulation for constructing Yang-Mills theories from the incorporation of non-linearities to the Maxwell's theory of electromagnetism. Our formulation is strongly based on Noether's Theorem and aims to show how the introduction of non-linearities can turn a global invariance into a local symmetry as the more general Yang-Mills type of symmetry.

\section{Introduction}

Yang-Mills Theories [1, 2, 3, 4] play an important hole on the description of both strong and weak interactions, being one of the most successful pillars of Fundamental Interactions. Nowadays electromagnetic and gravitational interactions can also be systematically described by the mechanisms of this class of field theories [5, 6, 7]. Here we develop a different approach to derive from classical Maxwellian electrodynamics a genuine classical Yang-Mills theory by using Noether Theorem and by introducing non-linearities to the Maxwellian regime. We start from a global symmetry and end up with a genuine Yang-Mills model having a local symmetry as the ultimate symmetry of the problem. In section 2 , we briefly review Maxwell's Electrodynamics in covariant notation and also explore briefly the concept of internal degrees of freedom and their correspondence to a colored electrodynamics. In section 3 we discuss a non-linear Yang-Mills model that describes an electromagnetic theory extension that can lead to an unification with the electroweak theory. In section 4, we discuss the formulation of general non-linear Yang-Mills models by introducing a recursive procedure from which we introduce gradually the non-linearities to the Lagrangian of the theory and see how the physical quantities transform according to the present symmetries. In section 5, we derive the final Lagrangian of the theory and also introduce the concept of Lie-algebra-valued fields. We also extend our model to the case of a more general $S U(N)$-group. In section 6 , we discuss the symmetries present in our problem and show how the initial global symmetry of the theory becomes local and the more general symmetry of the Yang-Mills model.

\section{Classical Electrodynamics and Internal Degrees of Freedom}

To covariantly formulate a theory means that we are explicitly making the Theory invariant under Lorentz Transformations. According to the nomenclature itself, covariant expressions will keep their exact form after the action of boosts or of any kind of change of inertial referential. For electromagnetic theory in a covariant formulation we may define the field strength, $F^{\mu \nu}$, of the electric and magnetic fields as

$$
F^{\mu \nu}=\partial^{\mu} A^{\nu}-\partial^{\nu} A^{\mu}
$$

$A^{\mu}$ being the four-potential $A^{\mu}=\left(\frac{\phi}{c}\right) \vec{A}$, where $\phi$ is the ordinary electrical potential, $c$ is the speed of light and $\vec{A}$ is the ordinary magnetic potential in Euclidean coordinates. Maxwell's equations are then written as

$$
\partial_{\mu} F^{\mu \nu}=4 \pi J^{\nu}
$$

where $J^{v}=(c \rho, \vec{J})$ is the four-current, $\rho$ being the ordinary charge density and $\vec{J}$ the ordinary electric current in Euclidean coordinates. The electric current $J^{v}$ can be written as a manifestly covariant four-vector after we introduce the four-vector coordinates of the particle, $r^{\alpha}(\tau)$, that is a function of the particle proper time $\tau$, and after we integrate over the proper time including an appropriate delta function [8]

$$
J^{\nu}(x)=q c \int d \tau U^{\nu}(\tau) \delta^{(4)}[x-r(\tau)],
$$

where $U^{v}(\tau)$ is the four-velocity of the charge and in the inertial frame of reference we have that $U^{v}=(\gamma c, \gamma \vec{v})$ and $r^{v}=[c t, \vec{r}(t)]$.

In ordinary electrodynamics the fields are invariant under the so called local Abelian gauge transformations, which take the form

$$
A^{\prime} \mu A^{\mu}+\partial^{\mu} \alpha
$$




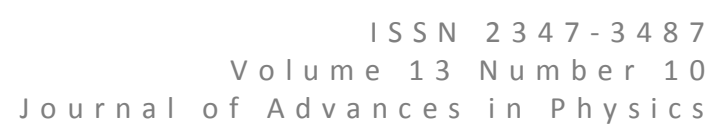

where $A^{\prime \mu}$ is the transformed potential and $\alpha$ is an arbitrary position-dependent parameter. In the case we want our theory to include internal degrees of freedom, such as a colored electrodynamics, there may be some second index, that we will label $a$ from now on, besides the space-time label $\mu$. Still preserving the $U(1)$ gauge transformation of ordinary electrodynamics the four potential $A_{a}^{\mu}$ will still transform under the $U(1)$ transformation such as

$$
A_{a}^{\prime \mu} \equiv A_{a}^{\mu}+\partial^{\mu} \alpha_{a}
$$

Note that only the space time index will be lowered or raised by the metric according to it covariant or contravariant nature, the index $a$ defined as being always a subscript label. Also the parameter $\alpha$ will carry the internal dof label $a$.

After the inclusion of the new internal symmetry the quantities will transform according to

$$
\left\{\begin{array}{c}
q_{a} \\
A_{a}^{\mu} \\
J_{a}^{\mu} \\
F^{\mu \nu}
\end{array}\right\}^{\prime} \equiv R\left\{\begin{array}{c}
q_{a} \\
A_{a}^{\mu} \\
J_{a}^{\mu} \\
F^{\mu \nu}
\end{array}\right\}
$$

where $R$ represents a rotation matrix related to a rotational symmetry that is an additional symmetry present in color electrodynamics. The more general rotational symmetry in the formulation of the Yang-Mills theory will be a $S U(N)$ transformation. Here we note that whereas in ordinary electrodynamics the electric charge $q$ is a unique quantum number, when we tackle the case of a colored electrodynamics the charge will become a vector $q_{a}$ whose number of components will be the number of internal dof introduced, that means, the number of colored charges. In a Yang-Mills formulation that takes into account ordinary electrodynamics gauge symmetry plus the internal degrees of freedom's rotational symmetry, fields are invariant with respect to the more general symmetry of the problem and the number of generators are equal to the number of independent fields of the theory. In a recent paper this restrict relation was extended to the notion of families of fields transforming under a same symmetry, what can probably led to an extension of QCD [9].

\section{Four Bosons Electrodynamics}

Group theory is a fundamental mathematical apparatus from where Yang-Mills' theoretical structure is based. It establishes that the number of potential fields coincide with the number of the group's generators, what defines the fields that take values on the group's algebra. By using the definition of the Lie algebra-valued fields, which will be discussed during the construction of this paper, we can expand the potentials as $A_{\mu}=A_{\mu a} G_{a}$, where $G_{a}$ is the group's generators.

From the years 1940 - 1960, several studies pointed on the direction of building electrodynamic models for bosonic carriers of spin-0 and spin-1 [10,11,12,13,14,15,16,17, 18, 19, 20,21]. The main purpose was to couple the photon to vectorial charged bosons. This was a preamble to the Electroweak Theory, whose unification took place definitely with the works by Salam-Glashow-Weinberg $[11,13,15,22,23,24,25,26]$ and the inclusion of the Higgs boson [27]. The inclusion of the Higgs mechanism and the spontaneous break of electroweak symmetry were decisive for eliminating several inconsistencies that were characterizing the models elaborated by that time.

On the idea of the Yang-Mills families [9] we just mentioned in section 2, a non-linear model for color electrodynamics' extension was introduced in previous works [28, 29], which is based in a system of four mediators for the electromagnetic interaction. Our alternative proposal consists on the electrical charge being carried by spin-0 and spin-1 particles, and considering the charge exchange it emerges the possibility of an electromagnetism that unifies the Maxwellian electrodynamics to the weak interaction based on four vectorial fields and without the non-Abelian symmetry. This quadruplet of vectorial bosons consists on the ordinary photon, a massive neutral photon and a dublet of charged photons. More specifically the mediators of the model can be considered as being the photon $A_{\mu}$ and the triplet $\left(\mathrm{W}^{ \pm}, \mathrm{Z}^{0}\right.$ ).

The fields corresponding to the four bosons of the model can be contracted with the SU(2) group's generators as

$$
B_{\mu a} \frac{\sigma_{a}}{2}=\frac{1}{2}\left(\begin{array}{cc}
B_{\mu 3} & B_{\mu 1}-i B_{\mu 2} \\
B_{\mu 1}+i B_{\mu 2} & -B_{\mu 3}
\end{array}\right)
$$

where we can perform the following identification for the triplet transforming under $S U(2)$ :

$$
\begin{aligned}
Z^{0} & \longrightarrow B_{\mu 3} \\
W^{+} & \longrightarrow B_{\mu 1}-i B_{\mu 2} \\
W^{-} & \longrightarrow B_{\mu 1}+i B_{\mu 2} .
\end{aligned}
$$

This four bosons model is derived basically by introducing non-linear couplings to the Maxwellian regime and is an example of a non-Linear Yang-Mills model for describing classical color electrodynamics. During the next sections we will explore the formulation of a fully general Yang-Mills theory coming from the introduction of non-linearities in Maxwell theory and by extensively using Noether's Theorem. 


\section{Introduction of the Non-Linearities}

The formulation of a color electrodynamics must be invariant under local Abelian gauge transformations and global color rotations. Here, however, we will follow the steps starting with a global symmetry that will be broken at the end, presenting a local symmetry as the more general symmetry of the Yang-Mills theory. We start with the case of a triplet of photons $A_{\mu a} \in S U(2)$ transforming as

$$
\delta A_{\mu a}=\epsilon_{a b c} \omega_{b} A_{\mu a}
$$

and the correspondent field strength varies accordingly to

$$
\delta F_{\mu \nu a}=\epsilon_{a b c} \omega_{b} F_{\mu \nu c}
$$

$\omega_{b}$ being a global parameter. The ordinary Maxwell electrodynamics' Lagrangian added with the internal dof label $a$ is then written as

$$
L_{1}=-\frac{1}{4} F_{\mu \nu a} F_{a}^{\mu \nu}
$$

Once this starting Lagrangian is invariant under the $S U(2)$ 's global transformation and the field equations resulting from it are still covariant, we can derive the four-current conserved in the model by variating the Lagrangian with respect to the potentials, finding

$$
j_{a}^{\mu}=\epsilon_{a b c} F_{b}^{\mu \nu} A_{\nu c}
$$

and getting the four current conservation, $\partial_{\mu} j_{a}^{\mu}=0$. The conserved four-current that was just derived will then be coupled to the original Lagrangian.

We include a further parameter $\beta$ to $j_{a}^{\mu}$ which does not afect the current conservation and will be relevant when we will identify the final system as a genuine Yang-Mills model. The conserved current becomes

$$
j_{a}^{\mu}=\beta \epsilon_{a b c} F_{b}^{\mu \nu} A_{\nu c}
$$

At this point we start the recursion procedure for the first time, by coupling the current of eq. (13) through the so called minimal prescription or minimal coupling

$$
\mathcal{L}_{2}=-\frac{1}{4} F_{\mu \nu a} F_{a}^{\mu \nu}-g j_{a}^{\mu} A_{\mu a}
$$

$g$ being the coupling parameter.

We now repeat the processes and variate the Lagrangian of eq. (14) with respect to the potential $A_{\mu a}$

$$
\delta_{A_{\mu a}} L_{2}=-\frac{1}{2} F_{a}^{\mu \nu}-g \beta \delta\left(\epsilon_{a b c} F_{b}^{\mu \nu} A_{\nu c} A_{\mu a}\right),
$$

leading to the following new equation of motion

$$
\partial_{\mu} F_{a}^{\mu \nu}-2 g \beta \epsilon_{a b c} \partial^{\mu}\left(A_{b}^{\mu} A_{c}^{\nu}\right)+2 g \beta \epsilon_{a b c} F_{b}^{\mu \nu} A_{\mu c}=0 .
$$

At this point we apply Jacobi identities and make use of the field's $S U(2)$ transformation described in eq. (9), getting, as a result, the following new conserved current

$$
j_{(2) d}^{\mu}=F_{a}^{\mu \nu} \epsilon_{a d e} A_{\nu a}-2 g \beta \epsilon_{a b c} \epsilon_{a d e} A_{b}^{\mu} A_{c}^{\nu} A_{\nu e},
$$

such that

$$
\partial_{\mu} j_{(2) d}^{\mu}=0
$$

We can now associate this conserved quantity with the conserved four-current of the Lagrangian $\mathcal{L}_{2}$. We also note that all non-linearities are being introduced through the conserved currents during this recursive procedure. At this point we remark that we can proceed with the recursive procedure as long as we wish, but if we couple the Lagrangian $\mathcal{L}_{2}$ to this next new conserved current, the extra term produced after the variation of the Lagrangian would not include total derivatives for the current, the conserved current remaining unchanged. This way it is important to note that we are not truncating the procedure, but it ends by itself. 


\section{Final Lagrangian and Lie-algebra-valued fields}

Following the reasoning we developed in section 4, the final Lagrangian results from coupling this last conserved current, $j_{(2) d}^{\mu}$, to $\mathcal{L}_{2}$, generating

$$
\begin{aligned}
\mathcal{L}_{3}= & -\frac{1}{4} F_{\mu \nu a} F_{a}^{\mu \nu}-g j_{(2) d}^{\mu} A_{\mu d} \\
= & -\frac{1}{4} F_{\mu \nu a}^{2}-g \epsilon_{a d b} F_{a}^{\mu \nu} A_{\nu b} A_{\mu d} \\
& +2 g^{2} \beta \epsilon_{a b c} \epsilon_{a d e} A_{b}^{\mu} A_{c}^{\nu} A_{\nu a} A_{\mu d} .
\end{aligned}
$$

Our final Lagrangian is then invariant under global $S U(2)$ transformations and from eq. (19) we see that the field equations will be non-linear: they are quadratic on $g$ and cubic on the potential $A$. Going again for the field equations by varianting the Lagrangian $\mathcal{L}_{3}$ with respect to the potential and by performing the reparametrization $g \rightarrow \frac{g}{2}$ and choosing $\beta \rightarrow-\frac{1}{2}$, we finally obtain for the first time a Yang-Mills Lagrangian

$$
\mathcal{L}_{Y M}=-\frac{1}{4}\left(F_{\mu \nu a}-g \epsilon_{a b c} A_{\mu a} A_{\nu b}\right)^{2}
$$

which is invariant under $S U(2)$ transformations.

At this point it is interesting from a formal point of view to introduce the concept of Lie-algebra-valued fields. Lets start by remembering $S U(2)$ 's algebra in the three dimensions representation. The generators of the group will take the following form

$$
\left(G_{a}\right)_{b c}=-i \epsilon_{a b c}
$$

and have commutation relations given by

$$
-i\left[G_{a}, G_{b}\right]=\epsilon_{a b c} G_{c}
$$

Defining

$$
\hat{F}_{\mu \nu a}:=F_{\mu \nu a}-g \epsilon_{a b c} A_{\mu a} A_{\nu b},
$$

and contracting with the generator $G_{a}$ we get

$$
\hat{F}_{\mu \nu a} G_{a}=F_{\mu \nu a} G_{a}+i g\left[A_{\mu b} G_{b}, A_{\nu b} G_{b}\right]
$$

such that we can define the following quantities

$$
\begin{aligned}
\hat{F}_{\mu \nu} & :=\hat{F}_{\mu \nu} G_{a} \\
F_{\mu \nu} & :=F_{\mu \nu} G_{a} \\
A_{\mu} & :=A_{\mu a} G_{a},
\end{aligned}
$$

which are the so called Lie-algebra-valued fields and, according to our definitions, we can write, more shortly,

$$
\hat{F}_{\mu \nu}:=\hat{F}_{\mu \nu}+i g\left[A_{\mu}, A_{\nu}\right] .
$$

The Yang-Mills formulation we have just derived is based on an invariance under a $S U(2)$ transformation. If we want to generalize our formulation from the $S U(2)$ invariance to a more general $S U(N)$ case we just have to introduce the $S U(N)$ structure constants in the place of the $S U(2)$ 's Levi-Civita symbol, which are the $S U(2)$ structure constants. We than have the following correspondence:

$$
\begin{array}{ll}
S U(2) & S U(N): a, b, c=1,2, \cdots, N \\
{\left[G_{a}, G_{b}\right]=i \epsilon_{a b c} G_{c}} & {\left[G_{a}, G_{b}\right]=i f_{a b c} G_{c}} \\
\left(G_{a}\right)_{b c}=i \epsilon_{a b c} & \left(G_{a}\right)_{b c}=-i f_{a b c} \\
\delta A_{\mu a}=i \epsilon_{a b c} \omega_{b} A_{\mu c} & \delta A_{\mu a}=i f_{a b c} \omega_{b} A_{\mu c}
\end{array}
$$

where for both groups of symmetry the generators are being represented in the adjoint representation.

Up to now the introduced symmetry is a global $S U(2)$ rotation, once it comes from the parameters $\omega_{b}$ being constant in all space-time. If we take the case of an infinitesimal transformation for the potential $A_{\mu a}$ we have 


$$
A_{\mu a} \rightarrow A_{\mu a}^{\prime}=\left(\mathrm{e}^{-i \omega_{d} G_{d}}\right)_{a b} A_{\mu b}
$$

Considering

$$
A_{\mu}=A_{\mu a} G_{a}
$$

and from the definition of the Lie algebra-valued fields we have that

$$
A_{\mu} \rightarrow A_{\mu}^{\prime}=\mathrm{e}^{-i \omega_{d} G_{d}} A_{\mu} \mathrm{e}^{+i \omega_{e} G_{e}},
$$

which in the case of the Lie algebra-valued field is a matrix transformation of similarity. Expanding the transformed potential $A_{\mu}^{\prime}$,

$$
\begin{aligned}
A_{\mu}^{\prime} & =\left(\mathbb{1}-i \omega_{d} G_{d}+\cdots\right) A_{\mu}\left(\mathbb{1}+i \omega_{e} G_{e}+\cdots\right) \\
& =A_{\mu}-i \omega_{c} G_{c} A_{\mu}+i \omega_{c} A_{\mu} G_{c}+\cdots
\end{aligned}
$$

the contraction with the generator $G$ becomes

$$
\begin{aligned}
A_{\mu a}^{\prime} G_{a} & =A_{\mu a} G_{a}+i \omega_{c}\left[G_{b}, G_{c}\right] A_{\mu b}+\cdots \\
& =A_{\mu a} G_{a}-\epsilon_{b c a} \omega_{c} A_{\mu b} G_{a}+\cdots,
\end{aligned}
$$

where we used that $\left[G_{b}, G_{c}\right]=i \epsilon_{b c a} G_{a}$, obtaining for the potential

$$
A_{\mu a}^{\prime}=A_{\mu a}+\delta A_{\mu a}+\cdots
$$

Finally for the field strength we end up with

$$
\hat{F}_{\mu \nu} \rightarrow \hat{F}_{\mu \nu}^{\prime}=\mathrm{e}^{-i \omega_{b} G_{b}} \hat{F}_{\mu \nu} \mathrm{e}^{+i \omega_{b} G_{b}}
$$

for the field transformation under the $S U(2)$ rotation.

\section{Discussing the Symmetries}

During the previous sections we saw that we can formulate a Yang-Mills theory by introducing non-linearities starting from a global symmetry. Now we will check the action of the non-linearities over the global symmetry and its breaking to generate the more general type of symmetry of the Yang-Mills model.

We start from the relation for the Lie-algebra-valued fields of eq. (26) that is valid for the more general case of a

$(\square)$ transformation. We now will follow the same recursive procedure but will work on a more general $\square \square$ ( $\square$ ) scenario. The beginning Lagrangian will them be

$$
\mathcal{L}_{(1)}=-\frac{1}{4} F_{a}^{\mu \nu} F_{\mu \nu a}
$$

On the other hand, in the potential transformation instead of the Levi-Civita symbol associated to $\square \square$ (2) operations there will be the structure constants $\square_{\square \square \square}$ referring to the $\square \square(\square)$ group. According to that the potential will transform such as

$$
\delta_{S U(N)} A_{\mu a}=f_{a b c} \omega_{b} A_{\mu c}
$$

Variating the Lagrangian in eq. (35) with respect to the potential and taking into account the potential $\square \square(\square)$ variation of eq. (36) we end up with the following conserved current

$$
j_{(1) a}^{\mu} \sim f_{a b c} F_{b}^{\mu \nu} A_{\nu c}
$$

whose variation, again respect to $\square \square(\square)$, is

$$
\delta_{S U(N)} j_{(1) a}^{\mu}=f_{a b c} \omega_{b} j_{(1) c}^{\mu} .
$$

Now we go back to the ordinary electrodynamics symmetry, namely a $\square$ (1) transformation. More specifically the $\square$ (1) variational will be characterized by

$$
\delta_{U(1)}=\partial_{\mu} \alpha_{a}
$$

and according to it, the field strength will variate as 

have that

$$
\delta_{U(1)} F_{\mu \nu a}=0 .
$$

Going back to the conserved current of eq. (37) and looking for how it varies according to the group $\square(1)$, we

$$
\delta_{U(1)} j_{(1) a}^{\mu} \sim f_{a b c} F_{b}^{\mu \nu} \partial_{\nu} \alpha_{a} \neq 0,
$$

what points out that the conserved current $\square(1) \square$ is not invariant under a local $\square(1)$ transformation.

Coupling the conserved current of eq. (37) to the original Lagrangian of eq. (35), according to the recursive procedure for formulating the Yang-Mills' final Lagrangian, we get a second Lagrangian $\mathcal{L}_{(2)}$ given by

$$
\mathcal{L}_{(2)}=-\frac{1}{4} F_{\mu \nu a}^{2}-\frac{g}{2} f_{a b c} F_{b}^{\mu \nu} A_{\nu c} A_{\mu c} .
$$

Now the conserved current of eq. (37) will be reparametrized to include the coupling constant of the Lagrangian such as

$$
j_{(1) a}^{\mu}=-\frac{g}{2} f_{a b c} F_{b}^{\mu \nu} A_{\nu c} .
$$

Following the same reasoning as before, when we were dealing with more restrict $\square \square(2)$ transformations, the way to introduce the non-linearities of the theory is by coupling the conserved currents to the Lagrangian trough this recursive procedure. However, now that we are tackling a more general transformation of the $\square \square(\square)$ group, is a remarkable point the fact that once $\square_{\square(1)} \square(1) \square \neq 0$ : in the exact moment that we introduce the current-photon coupling we loose the local $\square$ (1) symmetry typical of Maxwell's ordinary electrodynamics. That means that when we introduce the nonlinearity to the Maxwellian regime we loose the Abelian symmetry of electromagnetism. current

Coming back to the recursive procedure, we have for the second Lagrangian of eq. (42) the following conserved

$$
j_{(2) d}^{\mu} \sim f_{a d e} F_{a}^{\mu \nu} A_{\nu e}-2 g \beta f_{a b c} f_{a d e} A_{b}^{\mu} A_{c}^{\nu} A_{\nu e} .
$$

The variation of the conserved current of eq. (44) under both $\square \square(\square)$ and $\square(1)$ transformations is respectively

$$
\begin{aligned}
\delta_{S U(N)} j_{(2) d}^{\mu} & =f_{d e f} \omega_{e} j_{(2) f}^{\mu} \quad \text { and } \\
\delta_{U(1)} j_{(2) d}^{\mu} & \neq 0 .
\end{aligned}
$$

We are, than, able to build a third Lagrangian

$$
\begin{aligned}
\mathcal{L}_{(3)} & =-\frac{1}{4} F_{\mu \nu a}^{2}+\frac{g}{2} j_{(2) d}^{\mu} A_{\mu d} \\
& =-\frac{1}{4} \hat{F}_{\mu \nu a}^{2} \\
& =\mathcal{L}_{Y M} .
\end{aligned}
$$

As before, at this point the recursion stops by itself and $\mathcal{L}_{(3)}$ will be the final Lagrangian and a genuine Yang-Mills Lagrangian. We can also write the Yang-Mills Lagrangian in a more concise way if we look to the $\square \square(\square)$ group generators' properties, namely

$$
\begin{aligned}
\operatorname{tr}\left(G_{a} G_{b}\right) & =\left(G_{a}\right)_{c d}\left(G_{b}\right)_{d c}=\left(-i f_{a c d}\right)\left(-i f_{b d c}\right) \\
& =f_{a c d} f_{b c d} \\
& =\alpha \delta_{a b},
\end{aligned}
$$

such that

$$
\begin{aligned}
-\frac{1}{4} \operatorname{tr}\left(\hat{F}^{\mu \nu} \hat{F}_{\mu \nu}\right) & =-\frac{1}{4}\left(\hat{F}_{\mu \nu a} \hat{F}_{a}^{\mu \nu}\right) \operatorname{tr}\left(G_{a} G_{b}\right) \\
& =-\frac{1}{4} \hat{F}_{\mu \nu a} \hat{F}_{a}^{\mu \nu}
\end{aligned}
$$

Our final Yang-Mills' Lagrangian will then be written as

$$
\mathcal{L}_{Y M}=-\frac{1}{4} \operatorname{tr}\left(\hat{F}^{\mu \nu} \hat{F}_{\mu \nu}\right)
$$


This is a final way to write the Yang-Mills' formulated Lagrangian and we recall that the so found $\mathcal{L}_{(\square \square)}$ has lost its starting local $\square$ (1) symmetry, but still manifests the global $\square \square$ ( $\square$ ) symmetry because of the non-linearity that was introduced. The field strength will then transform under $\square \square(\square)$ such as

$$
\delta_{S U(N)} \hat{F}_{\mu \nu a}=f_{a b c} \omega_{b} \hat{F}_{\mu \nu c} .
$$

Now let us analyze the case of a finite transformation. In this finite case we can write

$$
\left(\hat{F}_{a}^{\mu \nu}\right)^{\prime}=R_{a b} \hat{F}_{b}^{\mu \nu}
$$

where the rotation matrix $\square$ is given by

$$
R_{a b}=\left(\mathrm{e}^{i \omega_{c} G_{c}}\right)_{a b}
$$

such that the similarity transformation of $\widehat{\square}_{\square \square}$ is given by

$$
\hat{F}_{\mu \nu}^{\prime}=R \hat{F}_{\mu \nu} R^{-1}
$$

By the same way, for the potential $\square \square$, we have for a finite rotation that

$$
\left(A_{a}^{\mu}\right)^{\prime}=R_{a b} A_{b}^{\mu} \Rightarrow A_{\mu}^{\prime}=R A_{\mu} R^{-1} .
$$

At this point we can ask the following question: would the global $\square \square(\square)$ symmetry be the ultimate and broader symmetry of the Yang-Mills' Lagrangian?

To answer that we come back to the infinitesimal $\square \square(\square)$ transformations case, where the field strength is given by

$$
\hat{F}_{\mu \nu a}=\partial_{\mu} A_{\nu a}-\partial_{\nu} A_{\mu a}-g f_{a b c} A_{\mu b} A_{\nu c}
$$

We will then propose that the parameter of the transformation $\square_{\square}$ is no longer global and we will add a second term $\square$ (1)type:

$$
\delta A_{\mu a}=f_{a b c} \omega_{b} A_{\nu c}+\frac{1}{g} \partial_{\mu} \omega_{a} .
$$

Now the parameter $\square_{\square} \equiv \square_{\square}(\square)$ has became local and the second term was added because we would like to have a local symmetry in order to eliminate the spin-0 contained in $\square_{\square \square}$. This way the Abelian symmetry of the Maxwellian regime that is lost during the introduction of the non-linearities leads naturally to the local $\square \square(\square)$ symmetry as it is characteristic of the Yang-Mills models. According to the new introduced symmetry we have that the variation of the field strength is given by

$$
\begin{aligned}
\delta \hat{F}_{\mu \nu a}= & \partial_{\mu}\left(f_{a b c} \omega_{b} A_{\nu c}+\frac{1}{g} \partial_{\nu} \omega_{a}\right)-\partial_{\nu}\left(f_{a b c} \omega_{b} A_{\mu c}+\frac{1}{g} \partial_{\mu} \omega_{a}\right) \\
& -g f_{a b c}\left(f_{b d e} \omega_{d} A_{\mu e}+\frac{1}{g} \partial_{\mu} \omega_{b}\right) A_{\nu a} \\
& =-g f_{a b c} A_{\mu b}\left(f_{c d e} \omega_{d} A_{\nu e}+\frac{1}{g} \partial_{\nu} \omega_{c}\right) \\
& =f_{a b c} \omega_{b} \hat{F}_{\mu \nu c} .
\end{aligned}
$$

Going back to the finite case of the more general transformations we have the field strength transforms under the similarity relation

$$
\left(\hat{F}_{\mu \nu}\right)^{\prime}=R \hat{F}_{\mu \nu} R^{-1}
$$

where the rotation matrix $\square$ is given by

$$
R=\mathrm{e}^{i \omega_{c} G_{c}}
$$

and the pair ( $\square_{\square}, \square_{\square}$ ) corresponds respectively to the parameter and the generator of the group.

Finally we see that the formulated Yang-Mills Lagrangian is invariant under a more general local $\square \square(\square)$ transformation, 


$$
\delta_{S U(N)_{\text {local }}} \mathcal{L}_{Y M}=0 .
$$

Analogously to the field strength, the potential $\square \square$ will transform under a local $\square \square(\square)$ transformation such as

$$
\delta_{S U(N)_{\text {local }}} A_{\mu a}=f_{a b c} \omega_{b} A_{\nu c}+\frac{1}{g} \partial_{\mu} \omega_{a},
$$

and in the finite case we have the general transformation

$$
A_{\mu}^{\prime}=R A_{\mu} R^{-1}+\frac{i}{g} R\left(\partial_{\mu} R^{\dagger}\right)
$$

that coincides exactly to the Yang-Mills formulation.

\section{Conclusions}

The main purpose of this work consisted on formulating a genuine Yang-Mills theory from extending Maxwell's electrodynamics with symmetry $\square(1)$ to a colored electrodynamics with internal degrees of freedom, transforming under a rotational symmetry consistent with the colored electrodynamics' quantum numbers. The transition between the ordinary electrodynamics and the derived Yang-Mills' model is through the inclusion of non-linearities coming from the conserved currents that are coupled to the Lagrangian of the theory through a recursive procedure that allows us to derive a final Lagrangian which is invariant with respect to the symmetries of the problem. We construct the theory by supposing the more general case of a $\square \square(\square)$ symmetry. We show that the ultimate symmetry of the model consists on the change from an initial global symmetry to a local one, causing the loss of the Maxwellian $\square$ (1) symmetry and turning our model into a genuine non-Abelian Yang-Mills theory.

We note that the derived Yang-Mills' Lagrangian takes into account the colored electrodynamics purely, but we can also extend the theory by adding new terms to the Lagrangian, as including other types of interactions end electromagnetic theory's extensions such as anisotropic terms, couplings with background fields and Higgs mechanism. From the Lagrangian we can extract Maxwell's equations, investigate the dispersion relations of the theory, for example, and look for anomalous phenomena such as bi or multi-refringence.

\section{Acknowledgments}

We kindly thank to Fancisco Figueiredo and Naiara Y. Klein for the so much relevant discussions and support during this work, and the group Professor Global [30] for the precious scientific content that was so important for this study. We also thank to the Brazilian agencies CAPES and CNPq for the financial support of this project.

\section{References}

[1] C. N. Yang and R. L. Mills. Conservation of isotopic spin and isotopic gauge invariance. Phys. Rev., 96:191- 195, Oct 1954.

[2] R. Shaw. The Problem of Particle Types and Other Con- tributions to the Theory of Elementary Particles. PhD thesis, Cambridge, UK, 1955.

[3] C. N. Yang. Selected Papers (1945-1980) of Chen Ning Yang: With Commentary. World Scientific, 2005.

[4] G. Hooft. 50 Years of Yang-Mills Theory. World Scientific, 2005.

[5] S. Deser. The limit of massive electrodynamics. Annales de I'I.H.P. Physique thorique, 16(1):79-85, 1972.

[6] S. Deser. Gravity from self-interaction in a curved background. Classical and Quantum Gravity, 4(4):L99, 1987.

[7] D G. Boulware, S Deser, and J H. Kay. Supergravity from self-interaction. 96:141-162, 041979.

[8] John David Jackson. Classical Electrodynamics. John Wiley \& Sons, 1975.

[9] Renato Doria. A new model for a non-linear electromagnetic model with self-interacting photons. Journal of Advances in Physics, 7(3):1840-1896, 2011.

[10] H. C. Corben and Julian Schwinger. The electromagnetic properties of mesotrons. Phys. Rev., 58:953-968, Dec 1940.

[11] A. Komar and Abdus Salam. Renormalization problem for vector meson theories. Nuclear Physics, 21:624 - 630, 1960.

[12] T. D. Lee and C. N. Yang. Theory of charged vector mesons interacting with the electromagnetic field. Phys. Rev., 128:885-898, Oct 1962.

[13] Abdus Salam. Renormalizable electrodynamics of vector mesons. Phys. Rev., 130:1287-1290, May 1963.

[14] K. H. Tzou. II Nuovo Cimento, 33:286, 1964.

[15] Abdus Salam and Robert Delbourgo. Renormalizable electrodynamics of scalar and vector mesons. ii. Phys. Rev., 
135:B1398-B1427, Sep 1964.

[16] Giorgio Velo and Daniel Zwanzinger. Noncausality and other defects of interaction lagrangians for particles with spin one and higher. Phys. Rev., 188:2218-2222, Dec 1969.

[17] Harmon Aronson. Spin-1 electrodynamics with an electric quadrupole moment. Phys. Rev., 186:1434-1441, Oct 1969.

[18] Bert Schroer. Peculiarities of massive vector mesons and their zero mass limits. The European Physical Journal C, 75(8):365, Aug 2015.

[19] M. Kuroda, J. Maalampi, K.H. Schwarzer, and D. Schild- knecht. Non-standard self-interactions of the weak vector bosons and their phenomenological implications. Nuclear Physics B, 284:271 - 298, 1987.

[20] I. Kuss and D. Schildknecht. Discovering non-abelian weak couplings and an anomalous magnetic dipole moment of the $\mathrm{w} \pm$ at lep 2. Physics Letters B, 383(4):470 - 474, 1996.

[21] M. Bilenky, J.L. Kneur, F.M. Renard, and D. Schildknecht. Trilinear couplings among the electroweak vector bosons and their determination at lep2. Nuclear Physics B, 409(1):22 - 68, 1993.

[22] Abdus Salam. Weak and electromagnetic interactions. In Nils Svartholm, editor, Elementary particle theory, pages 367-377. Almquist \& Wiksell, 1968.

[23] S. Kamefuchi, L. O'Raifeartaigh, and Abdus Salam. Change of variables and equivalence theorems in quantum field theories. Nuclear Physics, 28(1):529 - 549, 1961.

[24] Steven Weinberg. A model of leptons. Phys. Rev. Lett., 19:1264-1266, Nov 1967.

[25] H. Georgi and S.L. Glashow. Unity of all elementary particle forces. Phys. Rev. Lett., 32(438), Feb 1974.

[26] Sheldon L. Glashow. Partial-symmetries of weak interactions. Nuclear Physics, 22(4):579 - 588, 1961.

[27] Peter W. Higgs. Broken symmetries and the masses of gauge bosons. Phys. Rev. Lett., 13:508-509, Oct 1964.

[28] S. Machado Moreira. Efeitos Eletrodinâmicos Não-Lineares e Sistemas Quânticos Abertos. PhD thesis, Centro Brasileiro de Pesquisas Físicas, BRA, 2017.

[29] J. Chauca, R. Doria, and I. Soares. Four bosons electromagnetism. Journal of Advances in Physics, 10(1):2605, 2015.

[30] S. A. Dias. Professor global class notes. http://www.professorglobal.com.br. Accessed: 2016. 